\title{
Effects of Non-Standard Interactions in the MINOS Experiment
}

\author{
Tommy Ohlsson \\ Department of Theoretical Physics, School of Engineering Sciences, Royal Institute of Technology (KTH) - \\ AlbaNova University Center, Roslagstullsbacken 21, SE-106 91 Stockholm, Sweden \\ Royal Swedish Academy of Sciences (KVA), P.O. Box 50005, SE-104 05 Stockholm, Sweden
}

\begin{abstract}
In this talk, we investigate the effects of non-standard interactions (NSI) in the MINOS experiment based on a full three-flavor neutrino oscillation framework simulation. The simulation was performed using the GLoBES software. The results indicate that the allowed region in the $\sin ^{2}\left(2 \theta_{23}\right)-\Delta m_{31}^{2}$ plane is extended due to NSI effects, that there is a degeneracy between the leptonic mixing angle $\theta_{13}$ and the NSI parameter $\varepsilon_{e \tau}$, and that MINOS can put a lower upper bound on $\sin ^{2}\left(2 \theta_{13}\right)$ than $\mathrm{CHOOZ}$ only for small values of $\left|\varepsilon_{e \tau}\right|$.
\end{abstract}

Keywords: neutrino oscillations, non-standard interactions, the MINOS experiment PACS: $13.15 .+\mathrm{g}, 14.60 . \mathrm{Lm}, 14.60 . \mathrm{Pq}$

\section{INTRODUCTION}

Neutrino oscillation physics is the leading description for neutrino flavor transitions. However, sub-leading mechanisms could be responsible for smaller parts of these transitions. One example of such sub-leading mechanisms is non-standard interactions (NSI), which is the topic of this talk where we study the NSI impact on the sensitivity of the MINOS experiment. Furthermore, the talks by Sugiyama and Ota are devoted to NSI. This talk is based upon Ref. [1], which was done in collaboration with Mattias Blennow and Julian Skrotzki. The outline of this talk is the following: First, we introduce neutrino oscillations with NSI. Second, we give the simulation details of MINOS. Third, we present the outcome of the simulation. Finally, we make a summary and draw our conclusions.

\section{NEUTRINO OSCILLATIONS WITH NON-STANDARD INTERACTIONS}

In flavor basis, the effective three-flavor Hamiltonian describing neutrino oscillations in matter is given by

$H_{\mathrm{fl}}=\frac{1}{2 E} U\left(\begin{array}{ccc}0 & 0 & 0 \\ 0 & \Delta m_{21}^{2} & 0 \\ 0 & 0 & \Delta m_{31}^{2}\end{array}\right) U^{\dagger}+\left(\begin{array}{ccc}V & 0 & 0 \\ 0 & 0 & 0 \\ 0 & 0 & 0\end{array}\right)$,

where $E$ is the neutrino energy, $U=U\left(\theta_{12}, \theta_{13}, \theta_{23}, \delta\right)$ is the leptonic mixing matrix, and $V=\sqrt{2} G_{F} N_{e}$ is the effective matter potential. Here $\theta_{12}, \theta_{13}, \theta_{23}, \delta, \Delta m_{21}^{2}$, and $\Delta m_{31}^{2}$ are the six usual standard neutrino oscillation
TABLE 1. Present bounds on NSI parameters.

\begin{tabular}{lll}
\hline$\left|\varepsilon_{e e}\right|<\mathscr{O}(1)$ & $\left|\varepsilon_{e \mu}\right|<0.010$ & $\left|\varepsilon_{e \tau}\right|<\mathscr{O}(1)$ \\
$\left|\varepsilon_{\mu \mu}\right|<0.017$ & $\left|\varepsilon_{\mu \tau}\right|<0.013$ & $\left|\varepsilon_{\tau \tau}\right|<\mathscr{O}(1)$ \\
\hline
\end{tabular}

parameters, $G_{F}$ is the Fermi coupling constant, and $N_{e}$ is the electron number density.

Non-standard interactions are interactions between neutrinos and fermions in matter (i.e., $u, d$, and $e$ ) not present in the Standard Model, of which some can have an additional effect on neutrino oscillations. The effective NSI parameters are

$$
\varepsilon_{\alpha \beta}=\sum_{f=u, d, e}\left(\varepsilon_{\alpha \beta}^{f f L}+\varepsilon_{\alpha \beta}^{f f R}\right) \frac{N_{f}}{N_{e}} .
$$

Now, if NSI are included, the effective Hamiltonian becomes

$$
\begin{aligned}
H_{\mathrm{fl}}= & \frac{1}{2 E} U\left(\begin{array}{ccc}
0 & 0 & 0 \\
0 & \Delta m_{21}^{2} & 0 \\
0 & 0 & \Delta m_{31}^{2}
\end{array}\right) U^{\dagger} \\
& +V\left(\begin{array}{ccc}
1+\varepsilon_{e e} & \varepsilon_{e \mu} & \varepsilon_{e \tau} \\
\varepsilon_{e \mu}^{*} & \varepsilon_{\mu \mu} & \varepsilon_{\mu \tau} \\
\varepsilon_{e \tau}^{*} & \varepsilon_{\mu \tau}^{*} & \varepsilon_{\tau \tau}
\end{array}\right) .
\end{aligned}
$$

Thus, the NSI are described by six additional parameters $\varepsilon_{e e}, \varepsilon_{e \mu}, \varepsilon_{e \tau}, \varepsilon_{\mu \mu}, \varepsilon_{\mu \tau}$, and $\varepsilon_{\tau \tau}$. The present bounds on the NSI parameters are presented in Table $1[2,3]$. Using these upper bounds, we observe that $\varepsilon_{e \mu}, \varepsilon_{\mu \mu}$, and $\varepsilon_{\mu \tau}$ can safely be neglected. What constraints could be put on $\varepsilon_{e e}, \varepsilon_{e \tau}$, and $\varepsilon_{\tau \tau}$ by future experiments?

Note that in Ref. [4] NSI with MINOS and atmospheric neutrino data have been investigated, whereas in 
TABLE 2. Standard and NSI parameters.

\begin{tabular}{cc}
\hline Standard parameters & NSI parameters \\
\hline $\sin ^{2}\left(2 \theta_{12}\right)=0.8$ & $\varepsilon_{e e}=0$ \\
$\sin ^{2}\left(2 \theta_{13}\right)=0.07$ or 0 & $\varepsilon_{e \mu}=0$ \\
$\sin ^{2}\left(2 \theta_{23}\right)=1$ & $\varepsilon_{e \tau}=0$ \\
$\Delta m_{21}^{2}=\left(7 \cdot 10^{-5}\right) \mathrm{eV}^{2}$ & $\varepsilon_{\mu \mu}=0$ \\
$\Delta m_{31}^{2}=\left(2.74 \cdot 10^{-3}\right) \mathrm{eV}^{2}$ & $\varepsilon_{\mu \tau}=0$ \\
$\delta=\pi / 2$ & $\varepsilon_{\tau \tau}=0$ \\
\hline
\end{tabular}

our work we will consider NSI with MINOS neutrino data only. Especially, the $v_{\mu} \rightarrow v_{e}$ appearance channel will have implications for $\theta_{13}$ and $\varepsilon_{e \tau}$.

Next, we discuss a two-flavor neutrino limit for NSI, which is achieved by making the replacements $\Delta m^{2} \rightarrow$ $\Delta m_{31}^{2}, \theta \rightarrow \theta_{23}$, and $V \rightarrow-\varepsilon_{\tau \tau} V$ in the standard twoflavor neutrino oscillation framework. We obtain the following effective neutrino oscillation parameters

$$
\begin{aligned}
\sin ^{2}(2 \tilde{\theta}) & =\frac{\sin ^{2}\left(2 \theta_{23}\right)}{\xi^{2}}, \\
\Delta \tilde{m}^{2} & =\Delta m_{31}^{2} \xi,
\end{aligned}
$$

where

$$
\xi=\sqrt{\left[\frac{2 E V}{\Delta m_{31}^{2}} \varepsilon_{\tau \tau}+\cos \left(2 \theta_{23}\right)\right]^{2}+\sin ^{2}\left(2 \theta_{23}\right)} .
$$

Using the above equations for a fixed neutrino energy $E$, it is possible to choose $\varepsilon_{\tau \tau}$ such that $\Delta \tilde{m}^{2}=$ $\sin \left(2 \theta_{23}\right) \Delta m_{31}^{2}$ and $\sin ^{2}(2 \tilde{\theta})=1$ (i.e., maximal mixing). Therefore, we expect an NSI degeneracy in the $\sin ^{2}\left(2 \theta_{23}\right)-\Delta m_{31}^{2}$ plane.

\section{SIMULATION OF THE MINOS EXPERIMENT}

We have simulated the MINOS experiment with the General Long Baseline Experiment Simulator (GLoBES) [5]. For the simulation, we have used a running time of five years with $3.7 \cdot 10^{20}$ protons on target and a neutrino energy $E$ in the interval between $1 \mathrm{GeV}$ and $6 \mathrm{GeV}$. The neutrino oscillation and NSI parameters used for the simulation is given in Table 2. All NSI parameters are assumed to be real. In addition, note that in all simulations, a full numeric three-flavor neutrino oscillation framework was used.

\section{OUTCOME OF SIMULATION}

In Fig. 1, we plot the sensitivity limits in the $\sin ^{2}\left(2 \theta_{23}\right.$ $\Delta m_{31}^{2}$ plane ( 2 d.o.f.) for the combined appearance and

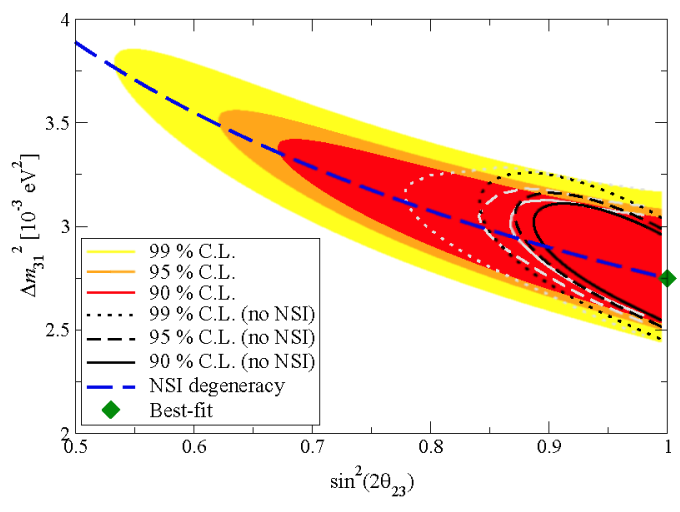

FIGURE 1. Sensitivity limits in the $\sin ^{2}\left(2 \theta_{23}\right)-\Delta m_{31}^{2}$ plane. This figure has been adopted from Ref. [1]
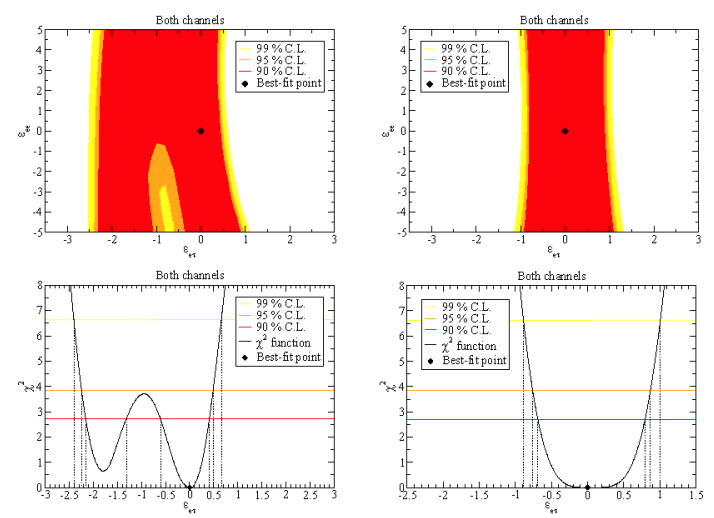

FIGURE 2. Sensitivity limits in the $\varepsilon_{e \tau}-\varepsilon_{e e}$ plane (left panel: $\sin ^{2}\left(2 \theta_{13}\right)=0.07$, right panel: $\left.\sin ^{2}\left(2 \theta_{13}\right)=0\right)$ and constraints on the NSI parameter $\varepsilon_{e \tau}$.

disappearance channels when allowing NSI compared with the sensitivity limits when not allowing NSI. We observe that including NSI will lead to a degeneracy in the $\sin ^{2}\left(2 \theta_{23}\right)-\Delta m_{31}^{2}$ plane (long-dashed curve). Similar results have been obtained in Ref. [4]. Next, in Fig. 2, we show an example of how constraints on the NSI parameters can be obtained. Here we study the NSI parameter $\varepsilon_{e \tau}$. Using the $\chi^{2}$-functions in the two lower panels in this figure, we can read off the numerical values of $\varepsilon_{e \tau}$. The result is given in Table 3 . In order to investigate the NSI effects on $\sin ^{2}\left(2 \theta_{13}\right)$, we can study the effective three-flavor mixing matrix element $\tilde{U}_{e 3}$, which is given

TABLE 3. Constraints on the NSI parameter $\varepsilon_{e \tau}$.

\begin{tabular}{lrr}
\hline $\sin ^{2}\left(2 \theta_{13}\right)$ & Constraint & Confidence level \\
\hline 0.07 & $-2.16<\varepsilon_{e \tau}<-1.31$ & $90 \%$ C.L. \\
& $-0.60<\varepsilon_{e \tau}<0.41$ & \\
\hline 0 & $-0.69<\varepsilon_{e \tau}<0.8$ & $90 \%$ C.L. \\
\hline
\end{tabular}




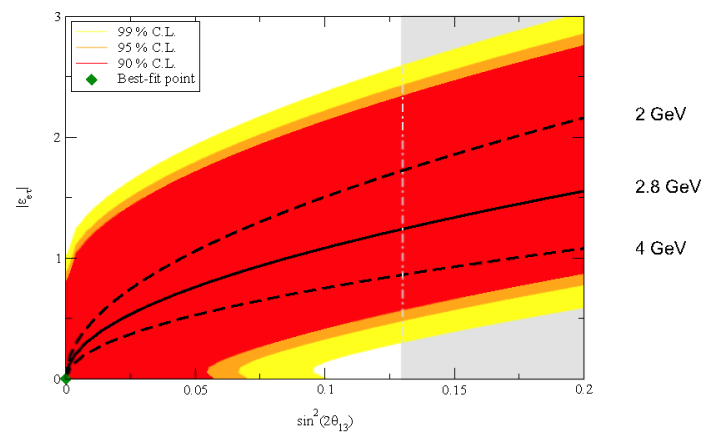

FIGURE 3. Sensitivity limits in the $\sin ^{2}\left(2 \theta_{13}\right)-\left|\varepsilon_{e \tau}\right|$ plane. This figure has been adopted from Ref. [1].

by [6]

$$
\tilde{U}_{e 3}=U_{e 3}+\varepsilon_{e \tau} \frac{2 E V}{\Delta m_{31}^{2}} \cos \left(\theta_{23}\right),
$$

where $\tilde{U}_{e 3} \propto \sin \left(\tilde{\theta}_{13}\right)$ and $U_{e 3} \propto \sin \left(\theta_{13}\right)$. Thus, using Eq. (6), it follows that, if $\tilde{U}_{e 3}=0$, then $\sin \left(\theta_{13}\right) \propto \varepsilon_{e \tau}$. In general, it holds that there are two main contributions to $\tilde{U}_{e 3}$, which are the contributions from $U_{e 3}$ and $\varepsilon_{e \tau}$. Thus, there will be a degeneracy along the curve $\tilde{U}_{e 3}=0$ for some fixed neutrino energy $E$. Finally, in Fig. 3, we present the sensitivity limits in the $\sin ^{2}\left(2 \theta_{13}\right)-\left|\varepsilon_{e \tau}\right|$ plane (1 d.o.f.) for the combined appearance and disappearance channels. Our numerical simulation show that $\left|\varepsilon_{e \tau}\right| \simeq$ 2.5 is needed in order to establish NSI if no further constraints can be put on $\theta_{13}$. However, in the optimistic case, the neutrino oscillation probability $P_{\mu e}$ will be so large that it cannot be described by the standard neutrino oscillation scenario, which implies the existence of NSI [7].

\section{SUMMARY \& CONCLUSIONS}

In summary, we have found that i) the allowed region in the $\sin ^{2}\left(2 \theta_{23}\right)-\Delta m_{31}^{2}$ plane is extended to smaller values of $\sin ^{2}\left(2 \theta_{23}\right)$ and larger values of the large mass squared difference $\Delta m_{31}^{2}$ if NSI effects are present, ii) there is a degeneracy between the leptonic mixing angle $\theta_{13}$ and the NSI parameter $\varepsilon_{e \tau}$, iii) the possible bounds on $\varepsilon_{e \tau}$ depend on the value of $\theta_{13}$, iv) MINOS can measure a better upper bound on $\sin ^{2}\left(2 \theta_{13}\right)$ than the $\mathrm{CHOOZ}$ experiment only for small values of $\left|\varepsilon_{e \tau}\right|$, and $\mathrm{v}$ ) MINOS could put a limit on $\left|\varepsilon_{e \tau}\right|$ if $\sin ^{2}\left(2 \theta_{13}\right)$ could be further constrained. In addition, our analytic results show that the disappearance channel $\left(v_{\mu} \rightarrow v_{\mu}\right)$ is mainly affected by $\varepsilon_{\tau \tau}$, whereas the appearance channel $\left(v_{\mu} \rightarrow v_{e}\right)$ is mainly affected by $\varepsilon_{e \tau}$.

\section{ACKNOWLEDGMENTS}

I would like to thank Mattias Blennow and Julian Skrotzki for useful collaboration that led to the publication [1] upon which this talk is based. In addition, I would like to thank the organizers of NuFact' 07 for the invitation.

This work was supported by the Royal Swedish Academy of Sciences (KVA) and the Swedish Research Council (Vetenskapsrådet), Contract No. 6212005-3588.

\section{REFERENCES}

1. M. Blennow, T. Ohlsson, and J. Skrotzki (2007), hep-ph/0702059.

2. S. Davidson, C. Peña-Garay, N. Rius, and A. Santamaria, $J$. High Energy Phys. 03, 011 (2003), hep-ph/0302093.

3. M. C. Gonzalez-Garcia, and M. Maltoni, Phys. Rev. D70, 033010 (2004), hep-ph/0404085.

4. A. Friedland, and C. Lunardini, Phys. Rev. D74, 033012 (2006), hep-ph/0606101.

5. P. Huber, M. Lindner, and W. Winter, Comput. Phys. Commun. 167, 195 (2005), hep-ph/0407333.

6. M. Blennow, T. Ohlsson, and W. Winter, Eur. Phys. J. C49, 1023-1039 (2007), hep-ph/0508175.

7. N. Kitazawa, H. Sugiyama, and O. Yasuda (2006), hep-ph/0606013. 